$\stackrel{W}{=}$

Global burnals Inc.

है

GLOBAL JOURNAL OF MEDICAL RESEARCH: K

INTERDISCIPLINARY

Volume 20 Issue 10 Version 1.0 Year 2020

Type: Double Blind Peer Reviewed International Research Journal

Publisher: Global Journals

Online ISSN: 2249-4618 \& Print ISSN: 0975-5888

\title{
Influence of Integration of Psychosomatic Concept on the Mental State of Elderly Patients in Emergency Ward
}

By Huijun Qi, Zhangshun Shen, Hui Guo, Qian Zhao \& Jianguo Li

Abstract- Objective: To explore the influence of the diagnosis and treatment mode based on thepsychological concept for the mental state of elderly patients in emergency ward.

Method: From January to March 2019,105 elderly patients in ward were selected as control group and given routine treatment mode;98 elderly patients as observation group, and the concept of psychosomatic medicine the was integrated intodiagnosis and treatment. The scores of SRQ, SAS, SDS and TCSQ at admission and discharge were compared between the two groups. The questionnaire survey of patients or family members at discharge were compared.

Results: Compared with the control group, at discharge the score of SRQ,SAS,SDS in the observation group were lower $(\mathrm{P}<0.05)$; the score of positive coping of TCSQwas higher than that in control group $(P<0.01)$, and the score of negative coping was lower than that in control group $(P<0.01)$. The family questionnaire at discharge showed attitudes, technical level, medication and satisfaction of the whole department in the observation group were higher than those in the control group. $\mathrm{P}<0.05$ ).

Keywords: psychosomatic medicine; emergency ward; elderly patients; mental state.

GJMR-K Classification: NLMC Code: WM 90

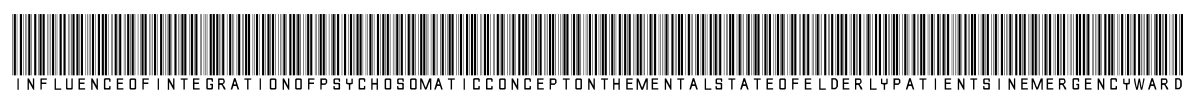

Strictly as per the compliance and regulations of:

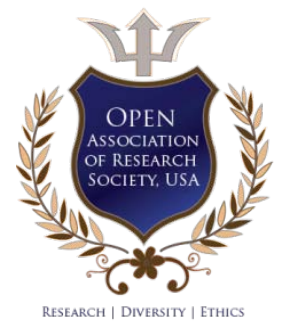

(c) 2020. Huijun Qi, Zhangshun Shen, Hui Guo, Qian Zhao \& Jianguo Li. This is a research/review paper, distributed under the terms of the Creative Commons Attribution-Noncommercial 3.0 Unported License http://creativecommons.org/licenses/by$\mathrm{nc} / 3.0 /$ ), permitting all non-commercial use, distribution, and reproduction in any medium, provided the original work is properly cited. 


\title{
Influence of Integration of Psychosomatic Concept on the Mental State of Elderly Patients in Emergency Ward
}

\author{
Huijun Qi ${ }^{\alpha}$, Zhangshun Shen $^{\circ}$, Hui Guo $^{\circ}$, Qian Zhao $^{\omega}$ \& Jianguo Li ${ }^{*}$
}

\begin{abstract}
Objective: To explore the influence of the diagnosis and treatment mode based on thepsychological concept for the mental state of elderly patients in emergency ward.
\end{abstract}

Method: From January to March 2019,105 elderly patients in ward were selected as control group and given routine treatment mode;98 elderly patients as observation group, and the concept of psychosomatic medicine the was integrated intodiagnosis and treatment.The scores of SRQ, SAS, SDS and TCSQ at admission and discharge were compared between the two groups. The questionnaire survey of patients or family members at discharge were compared.

Results: Compared with the control group, at discharge the score of SRQ, SAS, SDS in the observation group were lower $(\mathrm{P}<0.05)$; the score of positive coping of TCSQ was higher than that in control group $(P<0.01)$, and the score of negative coping was lower than that in control group $(P<0.01)$. The family questionnaire at discharge showed attitudes, technical level, medication and satisfaction of the whole department in the observation group were higher than those in the control group. $\mathrm{P}<0.05)$.

Conclusions: The model of diagnosis and treatment based on the concept of psychosomatic medicine can alleviate the psychological stress and bad mood of elderly patients in emergency ward, enable patients to respond positively to the disease and improve patient satisfaction.

Keywords: psychosomatic medicine; emergency ward; elderly patients; mental state.

\section{INTRODUCTION}

$\mathrm{P}$ sychosomatic medicine is an interdisciplinary subject that studies processes of physical, psychological and social interaction and their relevance to health and disease[1]. It makes up for the deficiency of biomedical model, helps to improve the treatment result and quality of life of patients, and provides more possibilities for the diagnosis and treatment of difficult medical problems.[2] As many countries are stepping into old age society, it is gradually recognized that elderly patients, like pediatric patients, have unique characteristics of diagnosis and treatment. The physical and mental of them are more fragile and sensitive than other patients, and they are

Author ¥: Emergency Department, Hebei General Hospital, Shijiazhuang, China.e-mail: lijg65@163.com more prone to fear, loneliness and helplessness. This psychological change may have an impact on the results of treatment and prognosis [3-4]. In recent years, the number of elderly patients admitted to emergency wards is increasing. they had higher negative psychological response. Many of them had anxiety, depression, Anxiety. Depression was one of the important factors affecting the prognosis of patients. How to apply psychosomatic medicine to emergency elderly patients was important social significance. Based on the routine diagnosis and treatment, this study combined coping skills, stress management and health education with the idea of psychosomatic medicine. And it's designed to relieve the emotional [5], And 98 elderly patients in emergency ward from April to June 2019 were prospectively observed and satisfactory results were obtained.

\section{Materials and Methods}

\section{a) Clinical data}

From January to March 2019,105 elderly patients were selected as control groupFrom April to June 2019,98 elderly patients were selected as the observation group. Inclusion criteria :(1) age >65 years; (2) Be conscious, Cognitive judgment and literacy skills, good communication; ${ }^{(3)}$. Length of stay $>3 \mathrm{~d}$; ${ }^{(4)}$ Exclusion of patients with malignant tumors, infectious diseases and major organ failure, All patients and their families signed informed consent.

Exclusion criteria: (1) had a history of mental illness, severe mental illness; (2) Termination or transfer of treatment; ${ }^{(3)}$ Systematic psychological intervention; ${ }^{(4)}$ Patients who refused to participate in the study. Comparison of general clinical data between the two groups was not statistically significant $(P>0.05)$ (see Table 1).

\section{b) Methods}

i. Control group: Using routine diagnosis and treatment process, medical staff regular rounds. Chief physician, deputy chief physician round 1-2 times a week, focus on the review of critical patients diagnosis and treatment plan, guide the handling of difficult cases, decide on major surgery or special examination and treatment, spot check the quality of 
medical care. The attending physicians conduct a round of rounds once a day to conduct a comprehensive tour of the patients under management, discuss the handling of critical and difficult cases and newly admitted patients, decide to consult, discharge or transfer, collect the patient's opinions, improve the work, and close the doctorpatient relationship. Residents should check twice a day and in the afternoon. Critical and unstable patients should be observed and treated at any time. Residents should also timely check the test results and special reports, analyze the condition, and report to the superior doctors in time.

The chief resident or night shift doctor shall patrol at night before the night shift. Monitor vital signs and explain the concerns of patients and their families, such as the necessity and safety of medical operation and treatment, guidance of medication, observation of adverse reactions, etc; give oral comfort to patients with bad mood to stabilize their emotions.

ii. Observation group: On the basis of routine diagnosis and treatment, the new mode is adopted with the concept of psychosomatic medicine. The contents are as follows

(1) Two comprehensive for doctor in charge: (1)They should fully understand the patient from head to toe to find problems and solve them,consult a specialistin timeif necessary; (1)They should have a comprehensive understanding from the nine aspects of "Clothing, food, shelter, transportation, sleep, psychology, family, environment and society" to collect patient information. The doctor in charge should understand the medical reimbursement systemto help the patients, and check the rational use of medicines, reduce the economic burdenof the family, and eliminate the worries of the family members.

(2) Cross-location of patients: The patients whom the same doctorin charge of are arranged in different rooms. When she/he visit her/his patients, Patients whom other doctorsare responsible for can also be visited and comforted, This way can increasethe frequency of contact and communication between doctors and patients and their families, and it will also increase the overall the ward care atmosphere for patients.

(3) Fixed frequency of communication: Rounds 5 times a day, Fixed time: come to work morning, noon off, come to workafternoon, afternoon off,night at 21:00 (chief resident or night shift doctor). Oral conversation, Concerned about the feelings of patients and their families, recordedin writing at any time the opinions and demands of patients and their families ,To enhance the trust and dependence of patients and their families on medical staff. [6]

(4) Mutual communication among patients: Encourage mutual understanding and communication among patients, to form a atmosphere of understanding, help and support.

(5) Psychological support: The elderly patients in the ward are sensitive and fragile, and the emotional anxiety affects the disease. Doctors are required to often encourage patients and family members to increase their confidence in treatment, help them to regulate the emotional, cultivate positive psychological to block the bad psychological factors[7].

\section{c) Clinical Data Collection}

(1) Psychological stress response: the psychological stress response questionnaire (SRQ) [8] was used to investigate, and after entering the stable condition (at admission) and leaving the ward (at discharge). The questionnaire included 3 dimensions, 28 entries, The three dimensions were somatic, emotional and behavioral responses, A negative score of $1 \sim 5$ for each entry. The higher the score, the more severe the response is. The internal consistency coefficient of the questionnaire is 0.910 .

(2) Mental state: The anxiety and depression were investigated by self-rating anxiety scale (SAS) and selfrating depression scale (SDS) at admission and discharge. SAS、SDS the scale contains 20 entries each, Grade 4 for each entry, SAS score $\geq 50$ for anxiety, SDS score $\geq 53$ with depression[9].

(3) The hospital satisfaction was investigated by patients and their family at discharge. The questionnairescale is same one for all the hospital.

\section{d) Statistical Analysis}

SPSS 23.0 software was used for statistical analysis. The measurement data consistent with normal distribution were represented by $\bar{x} \pm s$, and the comparison between groups was performed by independent sample $T$ test. The count data were expressed by percentage, and the comparison between groups was performed by $\chi^{2}$ test. Bilateral test was used, and $P<0.05$ indicated statistically significant differences.

\section{Results}

a) Comparison of baseline data between the observation group and the control groupgroups (Table 1)

There was no significant difference between the Observation and the Control group $(P>0.05)$ in general 
information such asgender, age, Marital status, Level of education, Medical Payment Method.

\section{b) Comparison of $S R Q$ scores in groups}

There was no difference between groups at admission in the scores of emotional response, somatic response and behavioral response. and which were lower in the observation group at discharge than those in the control group $(P<0.05)$ (Table 2$)$.

c) Comparison of SAS, SDS scores in groups.

There was no difference between groups at admission in the SAS, SDS score, which of the observation group was lower than that of the control group at discharge $(P<0.05)($ Table 3$)$;

\section{d) Comparison of TCSQ scores in groups}

There was no difference between groups at admission in the scores of positive and negative responses coping in the TCSQ and the scores of positive responses were higher in the observation group than those in the control group at discharge ,the scores of negative responses is opposite, there was difference between groups $(P<0.01)$ ( Table 4$)$

e) The questionnaire survey at discharge showed that 97 valid of 98 were distributed in the observation group. The control group received 104 valid questionnaires (total 105). The satisfaction of the observation group with the attitude, technical level, medication and the whole department were higher than that of the control group $(P<0.05)$ (see Table 5).

\section{Discussion}

The proportion of elderly people in the world is increasing day by day, whose physiological tissue structure and functional structure began to appear degenerative changes, and the function of respiration, circulation, digestion, endocrine and other systems was declined with the growth of age in elderly people, resulting in the phenomenon of weak and sick. Due to the weakness of the body, the decline of brain function, and the change of social status of the elderly, selfregulation imbalance, various negative emotions such as loneliness, depression, emptiness, nostalgia, anxiety and so on gradually become the dominant emotions in old age [3-4]. The incidence of depression and autism in the elderly has increased year by year, and studies have reported that among the elderly patients with physical health problems, accompanying mental problems has become an important factor that affeced diagnosis, treatment and prognosis [10] .More than 30\% of elderly patients with somatic diseases develop mental disorders associated with it. Among the elderly in Chongqing China, the detection rate of depression and anxiety was $57.2 \%$ and $40.8 \%$; , the proportion of subjective well-being was $43.8 \%$, which was at a lower level; loneliness was at a higher level, the proportion was $17.9 \%[11]$. We can also see this situation in this study. As a special group, their psychological problems should be paid more and more attention. How to provide better living conditions for the elderly to make their later life happy has attracted increasing attention.

Also, the number of elderly patients admitted to emergency wards is increasing, often with pessimistic, disappointed, anxious, fear and other emotional, this bad experience can easily affect the compliance of treatment and treatment effect. In the control group of this study, in the previous routine diagnosis and treatment mode, we paid attention to the observation of the patient's condition, and paid less attention to the psychological aspect. Although some personnel had carried out psychological care to elderly patients, but because of the single method, it cannot solve the problems encountered by patients, and it is easy to appear different degrees of stress (see table 2, table3, Table4).

The model of diagnosis and treatment, which is integrated with the concept of psychosomatic medicine, has been widely used in clinic in recent years. It has been reported that the this mode which integrates the concept of psychosomatic medicine can effectively alleviate the negative emotion of patients [12-13]. Psychological stress refers to the process of adaptation that people show through the whole psychological and physiological response after understanding and evaluating harmful, threatening and challenging to the outside world. Research reports, the patients in the emergency ward had a better physical response than the inpatients, and the ability to adopt coping behavior strategies is weak [14]. In this study, the elderly admitted in the emergency ward as the research object, They were paid more attention from head to toe, and to find problems and solve them,consult a specialistin timeif necessary, and to collect patient informationfrom the nine aspects of "Clothing, food, shelter, transportation, sleep, psychology, family, environment and society". In this way, their anxiety were relieved and worries solved from various aspects. In the other hand, a series of measures based on the concept of psychosomatic medicine: cross placement of patients, fixed frequency of communication, encouraging communication between patients, as well as the individualized psychological program, had been taken, and good results had been achieved, which reduced the psychological stress response caused by illness and hospitalization. The scores of emotional response, somatic response and behavioral response of SRQ at dischargein the observation group were lower than those in the control group $(P<0.05)$ (Table 2$)$ and the SAS/SDS score was lower than that of the control groupat discharge (Table 3).

It is very important to encourage communication, including the use of therapeutic 
communication and intervention among patients, medical staff and patients, patients and their families, so that elderly patients can accept the lonely time and regulate their bad emotions through their own behaviors [15].The diagnosis and treatment mode of psychosomatic medicine needs to pay attention to the role of social support factors in patients' psychological problems. Residents should do a good job in ideological work of family members, encourage patients in ward to communicate with each other, and establish a harmonious and friendly atmosphere, which has positive significance for relieving patients' negative emotions, and has a certain significance for their own disease treatment and prognosis [16]. In this study, score of TCSQD showed a higher positive response in the observation group at discharge than in the control group, patients were more positive in the face of disease than the control group $(\mathrm{P}<0.05)$ (table4). In addition, Implementation of the responsibility system, Which inspired a doctor's sense of responsibility, can better take patient needs as a starting point, provide better service for patients. It ensured the quality of the diagnosis and treatment mode integrated with the concept of psychosomatic medicine. Family members and patients discharged from hospital questionnaire survey showed that the observation group not only had a higher degree of satisfaction with doctor's attitude and the whole department $(P<0.05)$, but also had a higher degree of satisfaction with the doctor's technical level than the control group $(P<0.05)$ (table5). In fact, doctors were the same in two groups, result aboved showed that a good doctor-patient relationship could obtain the trust of patients' families and get better treatment feelings.

Health problem is the core for the elderly life. Many elderly patients have anxiety, depression mood, It is not only one of the important reasons affecting the prognosis of patients, but also one of the factors leading to doctor-patient contradictions. The application of psychosomatic medicine to emergency elderly patients makes up for the deficiency of biomedical model, has important social significance, or is conducive to improving the current tense doctor-patient relationship.

Conflict of interest: no conflict of interest for all authors

Author contribution: Huijun Qi: direct participation, and review of articles; Hui Guo: implementation of research and statistical analysis; Zhangshun Shen: collection, analysis/disclosure of data,; Qian Zhao: collection, analysis/disclosure of data; Jianguo Li: direct participation, guidance.

\section{References Références Referencias}

1. Lei Huang,Liming Cheng,Haisong Cui, et al. The enlightenment of psychosomatic competence training for doctors in western countries[J].] Chinese
Journal of Medical Education ,2019,39(2):153-160. DOI : 10.3760/cma.j.issn .1673-677 X.2019.02.012.

2. Fengquan $\mathrm{Xu}, \mathrm{Yu}$ Zheng,Linjie $\mathrm{Xu}$, et al. Impacts of Psychosomatic Treatment on Female Hormones in the Patients of Climacteric Depression [J].World Journal of Integrated Traditional and Western Medicine, 2017(07): 10-13. doi: 10.13935/j.cnki. sjzx.170702

3. Tao Zou, Shuqiao Yao. A Medicalization of Geriatric Life from Psychosomatic Medicine [J].] Medicine and Society, 2007, 20(4):13-14. DOI: 10.3870/j.issn. 1006-5563.2007.04.005.

4. Yin Pei, Jie Zhang, Jie Chen, et al. Analysis of consultation characteristics for elderly patients in the department of psychosomatic medicine in Hospital of TCM[J]. Chinese Journal of Geriatric Heart Brain and Vessel Diseases, 2012,14(02): 176-177. Doi:10.3969/j.issn.1009-0126.2012.02.019.

5. PanZhang. Problems in Nursing the Critical Elderly Kept Observation in Resuscitation Room and the Countermeasures[J]. Geriatrics \& Health Care, 2018, 24(6): 687-689. doi:10.3969/j.issn.10088296.2018. 06.035.

6. Fang Zhang, Cuixiang Li, Xiangqin Song, et al. Effects of structural psychological nursing on sense of uncertainty and adverse cardiovascular events in patients with $\mathrm{PCI}[\mathrm{J}]$.$] Chinese Journal of Modern$ Nursing, 2017, 23(20): 2615-2618. DOI:10.3760/ cma.j.issn.1674-2907.2017.20.010.

7. Jiaxin Qi, LiZhang, Ke Ma, et al. Effect of psychological intervention on anxiety and depression in patients with B type Stanford aortic dissection[J].] Journal of Vascular and Endovascular Vascular Surgery, 2016, 2(3): 232-235. DOI:10.19418/j.cnki.issn2096-0646. 2016.03.013.

8. Yang Li,Changxiang Chen. Application of Stanford Acute Stress Reaction Questionnaire in Trauma Fracture[J].] China Rehabilitation Theory and Practice, 2015, 21(6): 717-722. DOI: 10.3969 /j.issn.1006-9771.2015.06.017

9. Quanquan Duan, Li Sheng. Differential validity of SAS and SDS among psychiatric non-psychotic outpatients and their partners [J]. Chinese Journal of Mental Health, 2012, 26(009): 676-679. DOI: 10.3969/j.issn.1000-6729.2012.09.007.

10. Xin Yu. Geriatric Psychiatry [M], Beijing: Peking University Medical Press, 2008. 229238.ISBN:9787117120296.

11. Ying Xie, Xiaoyi Chen. on the Mental Health and Influencing Factors of the Elderly in Chongqing J] Chinese Journal of Gerontology, 2017(12): 30603062. DOI: 10.3969/ j.issn.1005-9202.2017.12.092.

12. Hong Zhang, Jianping Sun, Jing Zhang, et al. Structural group psychotherapy for the elderly 2 Effects of Self-transcendence in Type 2 
Diabetes[J].] Chinese Journal of Gerontology, 2017, 37(17): 4386-4389. DOI: 10.3969/ j.issn.1005-9202.2017.17.107.

13. Pan Li,Fei Yuan. Effect of Diagnosis and Treatment Model on Treatment Compliance and Rehabilitation of Paralysis Patients[J].] Chinese Practical Medicine, 2015, 10(5): 246 -247. DOI:10.14163/j.cnki.11-5547/r.2015.05.178.

14. Huizhen Cao, Jiangming Zhong, Xia Chen. Correlation analysis of stress and coping styles among patients in Emergency Observation Room[J].] Chinese Journal of Modern Nursing, 2017, 23 (14): 1833-1836. Doi: 10.3760/ cma.j.issn.1674-2907.2017.14.002.
15. Min Zhan. Effect of therapeutic communication and intervention on postoperative recovery, psychological stress and hope level of patients with breast cancer[J].] China Medical Herald, 2017, 14(10):177-180. http://d.wanfang data.com.cn/periodical/yycyzx201710045

16. Shu Wei. Effect of comprehensive nursing intervention on psychological and quality of life of patients with radiofrequency ablation of atrial fibrillation[J]] Hebei Medicine, 2015, 37(17): 2712-2714. DOI:10.3969/j.issn.10027386.2015.17 .056 .

Table 1: Comparison of General Data

\begin{tabular}{lccc}
\hline \multicolumn{1}{c}{ Project } & $\begin{array}{c}\text { Observation } \\
\text { Group98 } \\
\text { Example) }\end{array}$ & $\begin{array}{c}\text { Control } \\
\text { group105 } \\
\text { Example }\end{array}$ & $P$ Value \\
\hline Gender (male/female) & $54 / 44$ & $57 / 48$ & $P>0.05$ \\
Age $(\mathrm{x} \pm \mathrm{s})$ & $70.53 \pm 16.23$ & $70.10 \pm 15.93$ & $P>0.05$ \\
Marital status (married/widowed) & $88 / 10$ & $94 / 11$ & $P>0.05$ \\
Level of education (primary/ & $45 / 21 / 14 / 18$ & $48 / 22 / 16 / 19$ & $P>0.05$ \\
secondary/secondary/university) & & & \\
Medical Payment Method & $60 / 24 / 14$ & $64 / 28 / 13$ & $P>0.05$ \\
Medical insurance/NRCMS/Fund & & &
\end{tabular}

Table 2: Groups before and after intervention SRQ Score comparison score $(x \pm s)$

\begin{tabular}{|c|c|c|c|c|c|c|}
\hline \multirow{2}{*}{ Group n } & \multicolumn{2}{|c|}{ Emotional response } & \multicolumn{2}{|c|}{ Somatic response } & \multicolumn{2}{|c|}{ Behavioral response } \\
\hline & Admission & Discharge & Admission & Discharge & Admission & Discharge \\
\hline $\begin{array}{l}\text { Observation } \\
\text { Group }(n=98)\end{array}$ & $21.53 \pm 9.12$ & $16.24 \pm 6.12$ & $19.43 \pm 7.78$ & $14.32 \pm 5.14$ & $10.56 \pm 4.78$ & $6.21 \pm 3.25$ \\
\hline $\begin{array}{l}\text { Control group } \\
\quad(n=105)\end{array}$ & $21.28 \pm 8.75$ & $18.66 \pm 6.83$ & $1972 \pm 6.87$ & $16.91 \pm 5.38$ & $10.43 \pm 4.92$ & $8.02 \pm 3.46$ \\
\hline tValue & 0.132 & 1.770 & 0.187 & 2.335 & 0.127 & 2.558 \\
\hline$P$ Value & 0.447 & 0.040 & 0.426 & 0.011 & 0.450 & 0.006 \\
\hline
\end{tabular}

Table 3: Comparison of SAS and SDS scores in 2 groups $(\mathrm{x} \pm \mathrm{s}$,

\begin{tabular}{cccccc}
\hline \multirow{2}{*}{ Group $n$} & \multicolumn{2}{c}{ SAS Score } & & \multicolumn{2}{c}{ SDS Score } \\
\cline { 2 - 3 } \cline { 5 - 6 } & Admission & Discharge & & Admission & Discharge \\
\hline $\begin{array}{c}\text { Observation Group } \\
(\mathrm{n}=98)\end{array}$ & $42.67 \pm 7.82$ & $33.86 \pm 5.28$ & & $42.32 \pm 6.61$ & $36.28 \pm 6.17$ \\
$\begin{array}{c}\text { Control group } \\
(\mathrm{n}=105)\end{array}$ & $41.93 \pm 5.64$ & $36.12 \pm 5.69$ & & $43.05 \pm 7.32$ & $39.42 \pm 7.35$ \\
$\quad \begin{array}{l}t \text { Value } \\
P \text { Value }\end{array}$ & 0.181 & 1.953 & & 0.497 & 2.195 \\
\hline
\end{tabular}


Table 4: Comparison of TCSQ scores between the two groups before and after intervention (score)

\begin{tabular}{lccccc}
\hline \multirow{2}{*}{ Group $n$} & \multicolumn{2}{c}{ Positive response } & & \multicolumn{2}{c}{ Negative responses } \\
\cline { 2 - 3 } \cline { 6 - 6 } & Admission & Discharge & & Admission & Discharge \\
\hline $\begin{array}{l}\text { Observation } \\
\text { Group }(\mathrm{n}=98)\end{array}$ & $29.24 \pm 7.82$ & $37.76 \pm 58$ & & $32.32 \pm 7.62$ & $24.88 \pm 4.57$ \\
$\begin{array}{l}\text { Control group } \\
(\mathrm{n}=105)\end{array}$ & $30.10 \pm 7 . .64$ & $33.86 \pm 7.64$ & & $32.65 \pm 7.32$ & $30.42 \pm 4.85$ \\
$T$ Value & 0.160 & 2.765 & & 0.499 & 5.620 \\
$P$ Value & 0.450 & 0.003 & & 0.310 & 0.000 \\
\hline
\end{tabular}

Table 5: Results of Patient and Family Discharge Questionnaire [\%(M)]

\begin{tabular}{|c|c|c|c|c|c|c|c|c|}
\hline \multirow{2}{*}{$\begin{array}{c}\text { Satisfaction survey } \\
\text { content }\end{array}$} & \multicolumn{3}{|c|}{ Observation Group $(n=97)$} & \multicolumn{3}{|c|}{ Control group $(n=104)$} & \multicolumn{2}{|c|}{$\begin{array}{l}\text { Satisfaction } \\
\text { comparison }\end{array}$} \\
\hline & Satisfaction & General & $\begin{array}{c}\text { Not } \\
\text { satisfied }\end{array}$ & Satisfaction & General & $\begin{array}{c}\text { Not } \\
\text { satisfied }\end{array}$ & $\chi^{2}$ Value & $P$ values \\
\hline $\begin{array}{l}\text { Attitude towards the } \\
\text { bed doctor }\end{array}$ & $100(97)$ & $0(0)$ & $0(0)$ & $93.27(97)$ & $3.85(4)$ & $2.88(3)$ & 6.076 & 0.030 \\
\hline $\begin{array}{l}\text { Technical level of the } \\
\text { tube bed doctor }\end{array}$ & 98.96(96) & $1.04(1)$ & $0(0)$ & $91.35(95)$ & $6.73(7)$ & $1.92(2)$ & 5.795 & 0.037 \\
\hline Medication to the bed & $100(97)$ & $0(0)$ & $0(0)$ & $92.31(96)$ & $5.77(6)$ & $1.92(2)$ & 7.467 & 0.015 \\
\hline $\begin{array}{l}\text { For the entire } \\
\text { department }\end{array}$ & 98.96(96) & $1.04(1)$ & $0(0)$ & $91.35(95)$ & $5.77(6)$ & $2.88(3)$ & 5.789 & 0.041 \\
\hline
\end{tabular}

